\title{
Prediction and Detection of Epileptic Seizure
}

\author{
Mohammad Zavid Parvez \\ Centre for Research in Complex Systems (CRICS), School of Computing \& Mathematics \\ Charles Sturt University, Australia \\ Manoranjan Paul \\ Centre for Research in Complex Systems (CRICS), School of Computing \& Mathematics \\ Charles Sturt University, Australia
}

\begin{abstract}
Epilepsy is one of the common neurological disorders characterized by a sudden and recurrent malfunction of the brain that is termed "seizure", affecting around 65 million individuals worldwide. Epileptic seizure may lead to many injuries such as fractures, submersion, burns, motor vehicle accidents and even death. It is highly possible to prevent these unwanted situations if we can predict/detect electrical changes in brain that occur prior to onset of actual seizure. When building a prediction model, the goal should be to make a model that accurately classifies preictal period (prior to a seizure onset) from interictal (period between seizures when non-seizure syndrome is observed) period. On the hand, for the detection we need to make a model that can classify ictal (actual seizure period) from non-ictal/interictal period. This chapter describes the seizure detection and prediction techniques with its background, features, recent developments, and future trends.
\end{abstract}

Keywords: Seizure, Epilepsy, EEG, SVM, EMD, Preictal, Ictal, Interictal

\section{INTRODUCTION}

Seizure is a neurological disorder due to sudden surge of electrical activity in the brain caused by structural abnormalities of the brain, encephalitis, lack of oxygen in the brain, brain injury, tumor, and some sort of dysfunctionalities of the brain (Sanei et al. 2007). Epilepsy is characterized by spontaneously recurrent seizures (Parvez et al., 2012). More than 50 million (i.e., 1\% of world population) individuals are diagnosed with epilepsy (Santaniello et al., 2011). Approximately 5\% of whole populations are experienced a seizure in their life time (Netoff, et al., 2009). Epileptic patients have intractable seizures which are likely to increase damage to neural tissue. Epilepsy can be controlled by medication in the most cases. Epilepsy causes many injuries such as fractures, submersion, burns, accidents and even death. It is highly possible to prevent this unwanted situation if we correctly and timely predict epilepsy before the actual seizure onset.

In the brain, neurons exploit chemical reaction to generate electricity to control different bodily actions and this ongoing electrical activity can be recorded graphically which is popularly known as Electroencephalogram (EEG). EEG is well accepted tool for epileptic seizure prediction/detection that can measure the voltage fluctuations of the brain (Dorr, et al., 2007; Rosso et al., 2003; Esteller et al., 2004; Guttinger et al., 2005; and Tang et al., 2012). Feature extraction, analysis, and classification of EEG signals are still challenging issues for researchers due to the variations of the brain signals. Variations of EEG signals depend on different brain locations, number of channels, and different patterns of signals from different people. Another challenge for epileptic seizure detection/prediction from EEG signals is to get reasonable accuracy for real time applications. 
Generally we can describe a seizure detection technique by a number of steps such as noise/artefacts reduction using raw ictal, interictal, and non-seizure EEG signals, feature extractions, feature reduction, and classification using selected features. On the other hand, we can describe a seizure prediction technique by all steps of detection technique with two extra steps such as regularization of classification outcome and then decision making. The prediction technique normally classifies pre-ictal EEG signals to identify ictal signals from interictal or non-seizure EEG signals. As the similarity between pre-ictal and interictal signals are least compared to that between ictal and interictal signals, the prediction is more challenging compared to detection.

In this chapter we will discuss the fundamental steps (such as artifact removal, features extraction, features selection, classification, regularization of noisy output, and decision function, etc.) of the prediction and detection of epileptic seizure through the analysis of EEG signals. We will also discuss (i) the characteristics of EEG signals for healthy people and epileptic seizure patients, (ii) existing features extraction techniques with their advantages and limitations, (iii) existing classification techniques with their advantages and disadvantages, (iv) other challenging issues in near future for emerging technologies, and (v) future trend of research for high accuracy in detection and prediction.

\section{BACKGROUND}

Human brain processes sensory information received by external and internal stimuli. It controls movement and regulates cognitive functions such as thinking, learning, remembering, speaking, and decision making. The brain consists of perhaps 100 billion or more nerve cells called neurons (Craig et al., 2013). Every neuron has thousands of interconnections among them. Interconnected neurons are responsible for higher order thinking, complex behaviour, and it provides us with the ability to perceive, understand, and react to environmental events. The human brain is an organic electrochemical computer as neurons exploit chemical reactions to generate electricity. The electrochemical nature of neurons gives rise to our actions, our modes, and our behaviour. When a neuron becomes excited it passes electrochemical impulses incoming from the dendrites along the axon to communicate with other neurons in the brain (Neurology Applied, 2013) (see in Fig. 1). EEG is a non-invasive/invasive graphical record of ongoing electrical activity, which measures the changes of the electrical activity in terms of voltage fluctuations of the brain through multiple electrodes place on the scalp. In clinical contexts, the main diagnosis of EEG is to discover abnormalities of brain activities such as the epileptic seizure. Other clinical uses of EEG are in diagnosis of coma, brain death, encephalopathies, sleep disorder, etc. Moreover, the analysis of EEG signal has many other applications such as video quality assessment (Scholler et al., 2012), emotion recognition (Soleymani et al., 2012), and alcohol consumption measurement (Di et al., 2010), etc.

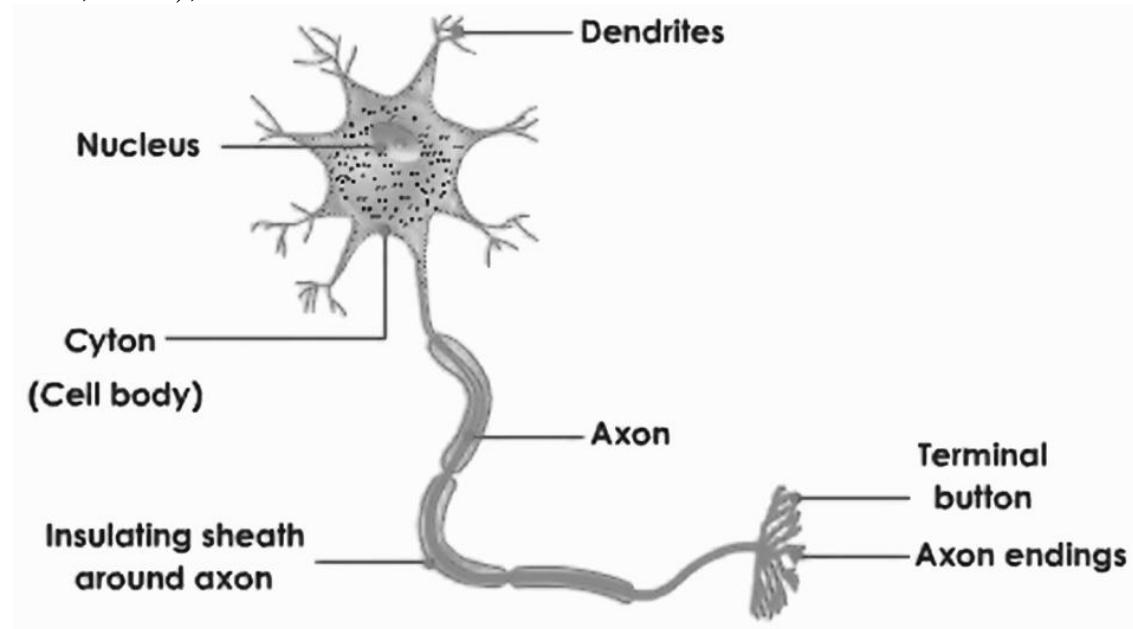

Figure 1. Structure of the neuron. Cell body contains nucleus and organelles where dendrites receive input signal, and axon conducts impulses away from the cell body. 
Seizure is simply the medical condition or neurological disorder in which too many neurons are excited in the same time caused by brain injury or by an imbalance of chemical in the brain that is characterized predominantly by unpredictable interruptions of normal brain function. Epilepsy is another medical condition characterized by spontaneously recurrent seizures. During the seizure period the brain cannot perform normal task as a result people may experience abnormal activities in movement, sensation, awareness, or behaviour. There are 1\% of total population ( around 50 million, WHO, 2013) in the worldwide affected by epileptic seizure (Netoff et al., 2009). 5\% of individuals are experience a seizure in their life time (Netoff et al., 2009). Approximate 30\% of seizure patients have intractable seizure which will increase the damange of the neural tissues (Zhou et al., 2013). It is possbile to stop seziure by medication in 70\% cases (Epilepsy Australia, 2014). Therefore, the detection of epileptic seizure plays important role for medical diagnosis of epilepsy. EEG recordings are very reliable in the diagnosis of epilepsy. EEG signals from an epileptic patient can be divided into five periods or stages (i) non-seizure period- no epileptic syndrome is visible, (ii) ictal period-actual seizure period, normally duration is 1 to 3 minutes or it could be more (iii) preictal period- 30 to 60 minutes before ictal period, (iv) post-ictal period- 30 to 60 minutes after ictal period, and (v) interictal period- period between postictal period to pre-ictal period of the immediate next ictal. Some portion of the interictal period, which does not have any epileptic syndrome, can be defined as a non-seizure period. Prediction and detection of seizures by analysing ictal, pre-ictal, and interictal could alert a patient of the next seizure and also could lead to better treatment and safety.

Two well-known epilepsy dataset are publicly available for the experiments; one is Bonn University Epilepsy dataset and another is Epilepsy Centre of the University Hospital of Freiburg Epilepsy dataset. Bonn University dataset consists of five subsets, each containing 100 single-channel EEG signals with 23.6 seconds duration where two subsets are scalp EEG and three subsets are intracranial EEG. Among them two subsets are taken from healthy volunteers (i.e., non-seizure dataset), two subsets are taken from seizure free intervals (i.e., interictal) and one subset is taken during seizure period (i.e., ictal) from five patients captured by 32 electrodes. Epilepsy Centre of the University Hospital of Freiburg dataset contains intracranial EEG recordings of 21 patients suffering from medically intractable focal epilepsy. The data was obtained by the Neurofile NT digital video EEG system with 128 channels, $256 \mathrm{~Hz}$ sampling rate, and 16 bit analogue-to-digital converter. Each patient has ictal and interictal file. The EEG signals containing epileptic seizures file with $50 \mathrm{~min}$ pre-ictal data. Later on, it is containing approximate 24 hours of EEG recoding without seizure activity. The ictal periods were determined based on identification of typical seizure patterns of experienced epileptologists.

Epileptic seizure detection technique can be done using different steps such as pre-processing (cleaning the dataset by removing various artifacts, line noise, etc.), features extraction, features selection and classification (see detailed explanation in section main focus of the article and corresponding figure at Fig. 4). A number of seizure detection techniques are already available to classify seizure and non-seizure EEG signals. Panda et al., 2010 computed various features like energy, entropy, and standard deviation by discrete wavelet transform and used support vector machine (SVM) as a classifier. Dastidar et al.,2007 applied wavelet transformation to decompose the EEG signals into different range of frequencies and three features, such as standard deviation, correlation dimension, and the largest Lyapunov exponent (quantifying the non-linear chaotic dynamics of the signals), are employed and different methods applied for classification. Ocak, 2008 proposed fourth level wavelet packet decomposition method to decompose the normal and epileptic EEG epochs to various frequency bands and then used genetic algorithm to find optimal feature subsets which maximize the classification performance. Polat et al., 2007 proposed two stage processes: first was feature extraction using first Fourier transform (FFT) and second was decision making using decision making classifier. The techniques have used Epilepsy Bonn University dataset which is small with 23.6 seconds duration and the classification accuracies were 91.2\% (Panda et al., 2010), 96.7\% (Dastidar et al., 2007), 98.72\% (Polat et al., 2007), and 98\% (Ocak, 2008).

Recently empirical mode decomposition (EMD) is proved to be an efficient transformation technique for EEG signal classification. Pachori, 2008 decomposed EEG signals into intrinsic mode 
function (IMF) using EMD and then computed mean frequency for each IMF to differentiate seizure and non-seizure EEG signals. Bajaj et al., 2012 extracted amplitude and frequency modulation bandwidth of IMFs as features using EMD. Among the existing contemporary techniques, Bajaj et al.'s technique is the latest and the best in terms of performance. They used least square SVM (LS-SVM) technique for the classification of seizure and non-seizure EEG signals using the Bonn University dataset with 23.6 second duration and obtained 98.0 to $99.5 \%$ accuracy using radial basis function (RBF) kernel and also obtained 99.5 to $100 \%$ accuracy using Morlet kernel. The Bonn University dataset with duration 23.6 second has seizure (i.e., ictal) and non-seizure signals which can be distinguished by their visual phenomena such as amplitude and changing rate of frequency (see in Fig. 2) where the non-seizure signal the amplitude is low and the frequency is high while the nature of seizure signal is totally reverse.

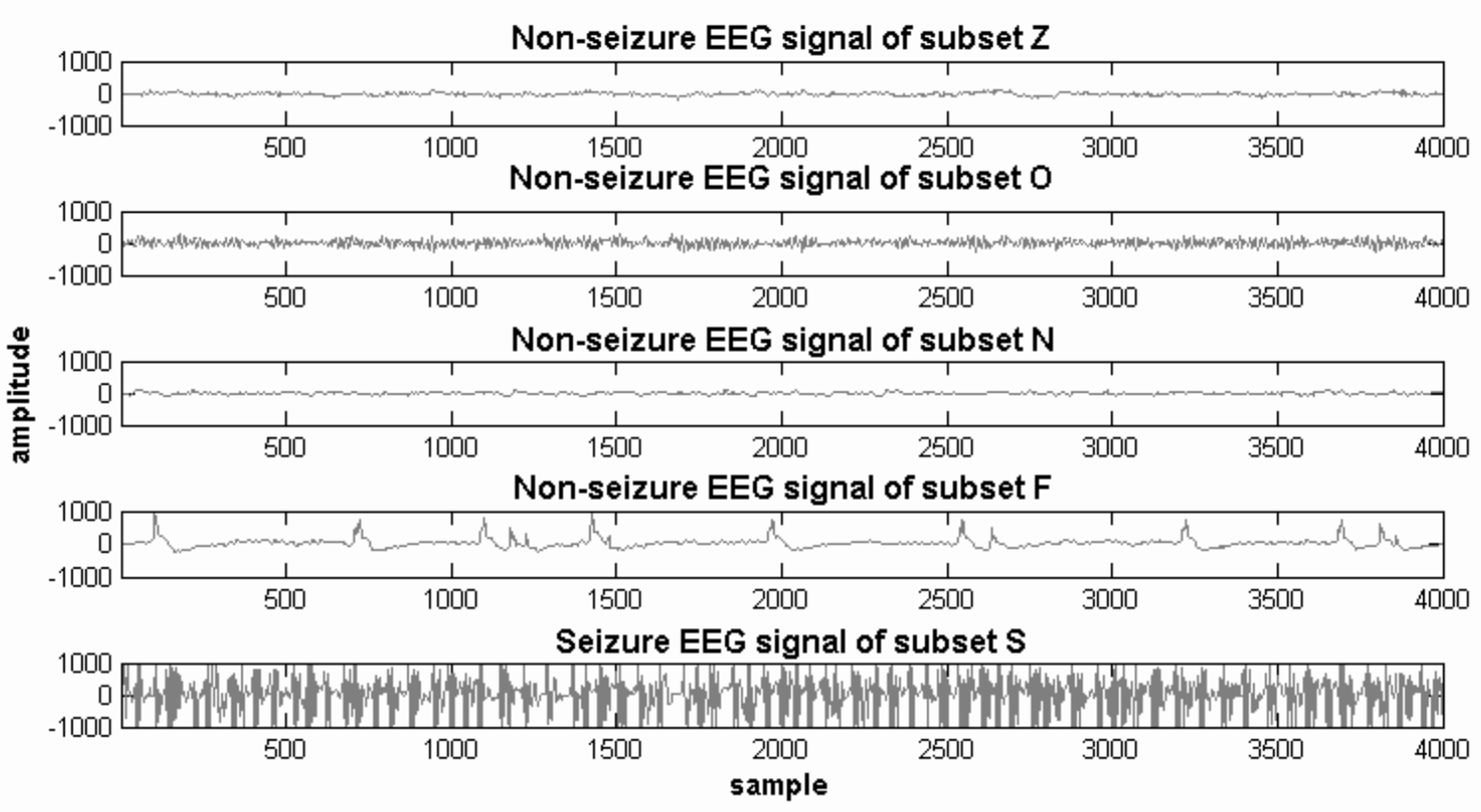

Figure 2. Seizure and non-seizure Bonn University dataset with 23.6 second duration, first four rows and fifth row indicated that the non-seizure and seizure data, respectively.

Parvez et al., 2013 proposed different feature extraction techniques, based on discrete cosine transformation (DCT), DCT -discrete wavelet transformation (DCT-DWT), and singular value decomposition (SVD), for ictal (seizure period) and interictal (period between seizures) EEG signals classification using Epilepsy Centre of the University Hospital of Freiburg dataset which contains 24 hour signals from each patient. Parvez et al., 2013 applied their techniques on two dataset (Bonn University and Epilepsy Centre of the University Hospital of Freiburg) and they got good classification accuracy for their techniques. They also demonstrated the non-abrupt phenomena (i.e., not easily distinguishable between ictal and interictal based on amplitude and frequency) of the ictal and interictal signal for the both cases of Frontal and Temporal lobe compared to the Epilepsy Centre of the University Hospital of Freiburg dataset shown in Fig. 3. Parvez et al., 2013 also shown that EEG signals from different locations exhibit different phenomenal activities for an ictal and interictal period. Thus, classification of ictal and interictal intracranial EEG signals are challenging compared to that of seizure (i.e., ictal) and non-seizure scalp EEG signals. Recetnly Parvez et al., 2014 and Parvez et al., 2015 show that exploiting temporal correlation and different transformations for feature extraction provides better seizure detection in ictal and interictal signal classification. 

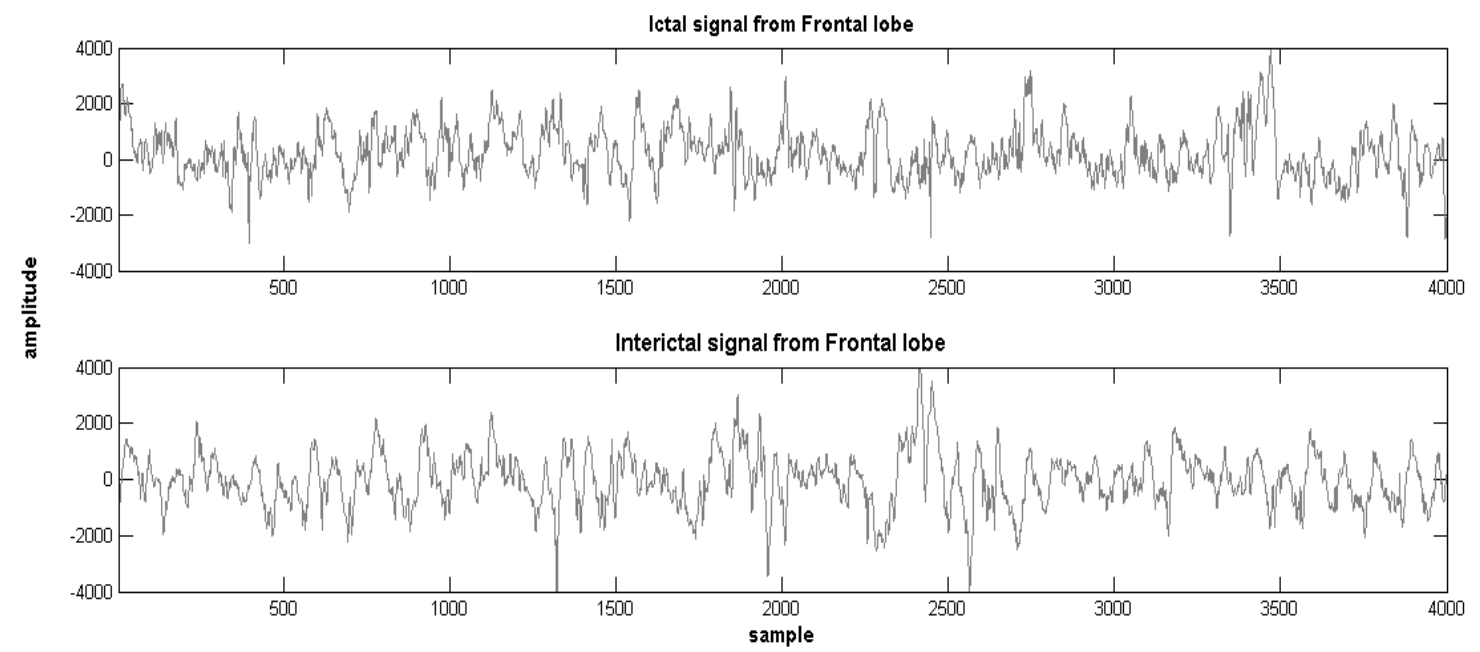

Figure 3. Ictal (Patient one, 7th data block, channel one) and interictal (Patient one, 95th data block, channel one) of Frontal lobe dataset from Epilepsy Centre of the University Hospital of Freiburg Epilepsy dataset.

Since the Bonn University dataset is small and perfectly cleaned, well defined seizure and nonseizure EEG signals, the accuracy of the above mentioned detection techniques perform very well that close to $100 \%$. However, in real scenario, we do not get cleaned signals; the signals might have different stages such as interictal, preictal, postictal, and ictal (i.e., seizure). Thus, when the above mentioned detection techniques are applied on the EEG signals with different stages, their performance is well below from the perfection e.g., $85 \%$ or less. Thus, detection of seizure with great accuracy is still a challenging issue.

Prediction of seizure is a difficult problem to provide a good trade-off between a high (close to $100 \%$ ) predictive capability of seizures (i.e., ictal) and a low (close to zero) false-positive rate (fpr) by analysing interictal and preictal signals assuming that ictal period will start immediate after preictal period. To predict an ictal signal we need to go through all steps of detection techniques (between interictal and preictal signals) and then we need to regularise the classification output using various techniques such as Kalman filter (Lefebvre et al., 2001), particle filter (Driessen et al., 2005), windowbased filtering (Netoff et al., 2009) and finally we need to apply a decision function for ultimate predicted output. Many methods have been proposed to predict epileptic seizure by classifying preictal and interictal EEG signals. They have employed univariate techniques (Rasekhi et al., 2013), eigenspectra of space delay correlation and covariance matrices (Williamsona et al., 2012), Hilbert-Huang transform (Duman et al., 2012), autoregressive modelling and least-squares parameter estimator (Chisci et al., 2010), bivariate features (Mirowski et al., 2009), spectral power from raw and bipolar time-differential signals (Park et al.,2011), and spike rate (Li et al., 2013).

A seizure prediction technique based on linear univariate features proposed by Rasekhi et al. provides $73.9 \%$ prediction accuracy with 11.1 false alarms per patient. Rasekhi et al. used their own dataset. Williamson et al. used spatiotemporal features and the experimental results show that the method provides $85 \%$ prediction accuracy with 0.8 false alarm per patient. They use 19 patients out of 21 patients from the benchmark dataset from Epilepsy Centre of the University Hospital of Freiburg. Chisci et al.'s used autoregressive model and support vector machines to predict seizure where they used 9 patients out of 21 patients from the same dataset. The prediction accuracy of the method is $100 \%$ with 10 false alarms per patient. Mirowski et al. proposed a seizure prediction technique based on bivariate features of EEG synchronization such as cross-correlation, nonlinear interdependence, dynamic entrainment, etc. They conducted experiments using 15 patients out of 21 patients. The method provides prediction accuracy $71 \%$ with no false alarm. Park et al. proposed a seizure prediction technique using linear features of 
spectral-power and non-linear classifier. They used 18 patients out of 21 patients. The method provides prediction accuracy $94.4 \%$ with 6.44 false alarms per patient on average. Li et al. applied spike rate using morphological filter to predict seizure. They obtained $75.8 \%$ prediction accuracy with 2.2 false alarms per patient using 21 patients. They used large of number of channels for seizure prediction. Therefore, they need more computational time and also need more sophisticated filtering technique to remove artifacts from all channels.

The prediction accuracies of the above mentioned seizure prediction techniques (Williamson et al., Mirowski et al., Li et al., Rasekhi et al.) are relatively low. On the other hand, the prediction accuracies of the seizure prediction techniques Chisci et al. and Park et al. are very good; however, the false alarm per patient is very high. Moreover, the above mentioned techniques (Williamson et al., Mirowski et al., Chisci et al. and Park et al. ) did not consider all patients of the Epilepsy Centre of the University Hospital of Freiburg dataset and they also used high number of channels for their approaches. Obviously using more channels requires more computational time and sometimes makes the systems more sensitive for artifacts which require sophisticated filtering. Comparison results of above mentioned methods are shown in Table 1. Prediction of epileptic seizure by analyzing EEG signals is a challenging task due to non-abruptness phenomena and inconsistency of the signals in different brain locations, patient-age, patient-sex, seizure-type, etc. Therefore, more research should be conducted for seizure prediction with better accuracy, low false alarm, and few numbers of channels and without sophisticated filtering technique to remove artifacts.

\begin{tabular}{|l|l|r|r|r|}
\hline \multicolumn{6}{|c|}{ TABLE 1 } \\
\hline \multicolumn{1}{|c|}{ COMPARISON RESUlTS WITH EXITING METHODS. } \\
\hline \multirow{4}{*}{ Methods } & \multicolumn{1}{c|}{ PA } & \multicolumn{1}{c|}{ FA } & Patients \\
\cline { 2 - 5 } & Williamson et al. & 85.0 & 0.80 & 19 \\
\cline { 2 - 5 } & Chisci et al. & 100 & 10.00 & 9 \\
\cline { 2 - 5 } & Mirowski et al. & 71.0 & 0.00 & 15 \\
\cline { 2 - 5 } & Park et al. & 94.4 & 6.44 & 18 \\
\cline { 2 - 5 } & Li et al. & 75.8 & 2.20 & 21 \\
\cline { 2 - 5 } & Rasekhi et al. & 73.9 & 11.10 & 10 \\
\cline { 2 - 4 } & Prediction accuracy=PA, False alarm= FA \\
\hline
\end{tabular}

\section{MAIN FOCUS OF THE ARTICLE}

The mechanism of producing epileptic seizure is still mysterious. The mechanism can be identified by combining features including firing rate, power spectral density and complexity analysis of the electrical signals of the human brain. Seizures (i.e. ictal) are characterized by large fluctuations of firing rate which is referring to dysfunctional regulation of neuronal activity.

Lots of research work has already made to the prediction of epileptic seizure based on real time analysis of EEG signals. Despite of remarkable advantage, results thus obtained are not sufficient for the realization of the real time signal analysis for the patient and cannot timely and reliably predict an oncoming epileptic seizure (Schelter et al., 2008). A major limitation of many proposed prediction techniques is their wide resort to demonstration of EEG signals that need offline processing. It is difficult for online processing to provide good prediction capability of seizure and low false positive rates due to increase number of EEG channels, and to introduce different aftifacts.

The term "Seizure Detection" and "Seizure Prediction" are significantly difference on the basis of characteristics of signals. In the case of detection, the seizure detection technique is not expected to identify the presence of a seizure until the characteristics of the seizure have appeared in the biologic signals being monitored. In the case of seizure prediction, the technique is expected to estimate seizures onset before they start. The difference can become confused when there is uncertainty about when the 
seizure starts and if the prediction technique is based on detecting a signal pattern that is consistently followed by a seizure. In this case the "prediction" method may be actually just "detecting" the seizure before its seizure onset.

EEG signal processing technique plays a significant role in prediction and detection of epileptic seizures. Recently, lots of research work has been devoted to the prediction and detection of epileptic seizures based on analysis of EEG signals. Properly prediction and detection of Epilepsy puts away the patients from fractures, submersion injuries, burns, vehicle accidents, etc. Therefore, it is significant to prevent epilepsy through correctly predict/detect preictal, ictal, and interictal stage to get accurate prediction/detection results. Feature extraction and classification of seizure detection can be processed offline but seizure prediction should be processed in real time. Basic block diagram of seizure detection and prediction are presented in Fig. 4 and Fig. 5 respectively.

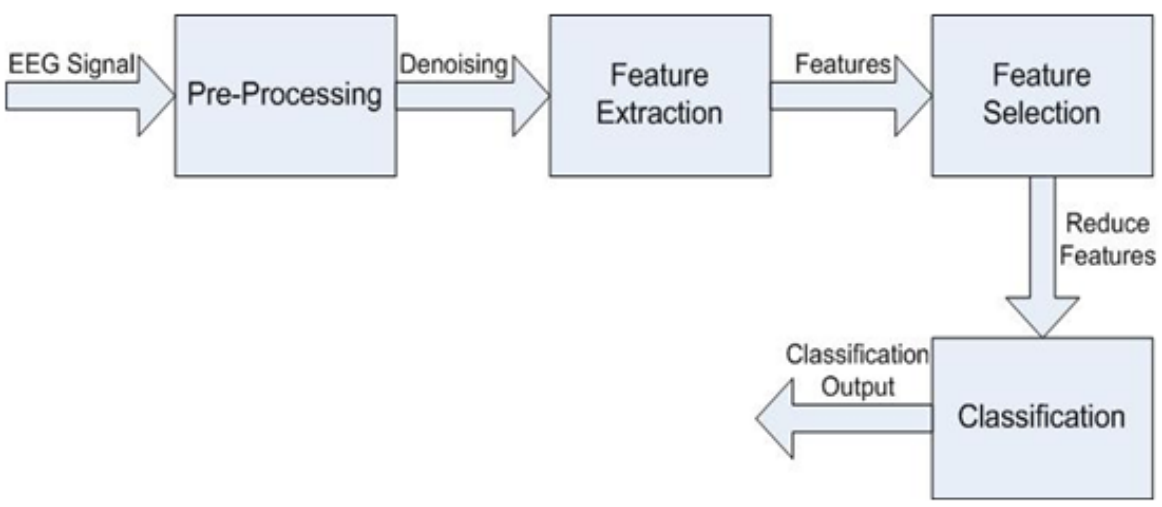

Figure 4. The basic seizure detection block diagram.

Before start the descripbtion of detection and prediction, we need to know about the human brain, EEG systems, and EEG waves. Later on, in the sub-sections will explain the basic steps of the detection and prediction techniques such as pre-processing, features extraction, features selection, classification, regularization and decision function.

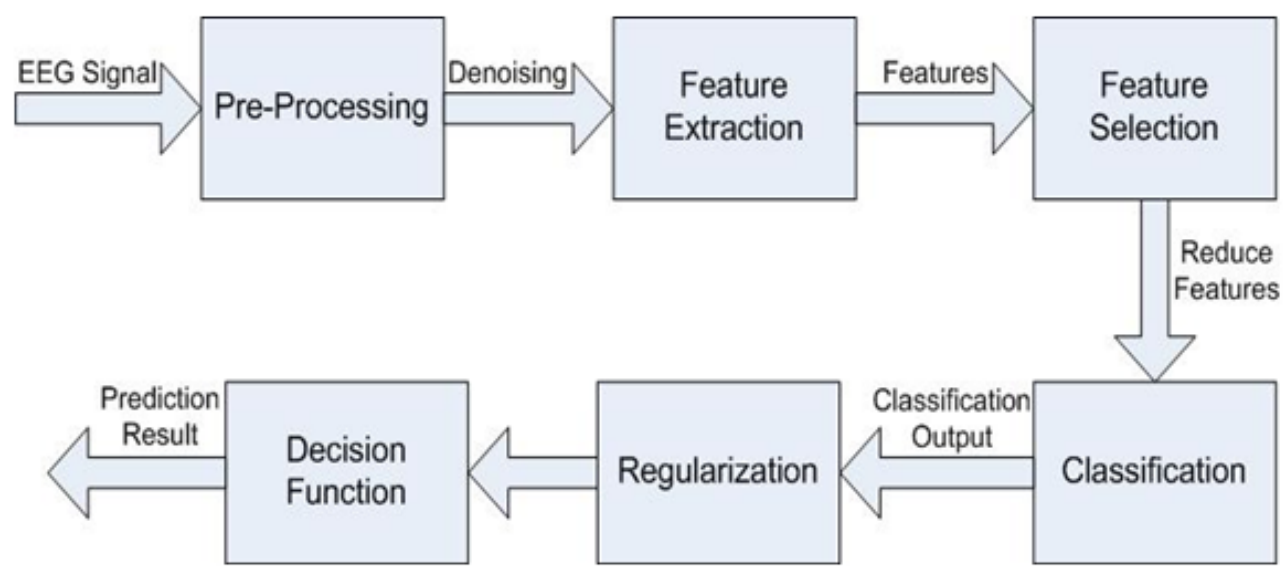

Figure 5. The basic seizure prediction block diagram.

\section{Human Brain}

Usually EEG signals are captured from the cerebral cortex of the human brain. Cerebral cortex can be subdivided into four lobes depending in its functions. The four lobes and their functionalities are explained below: 
Frontal lobe: It is responsible for execute functions, thinking, planning, organising, problem solving, emotions, behavior control, personality, etc. memory.

Temporal lobe: It is responsible for the primary organization of the sensory input and work as a

Parietal lobe: It can perceive the touch, pain, taste, pressure, and temparature and can understand the spelling, etc.

Occipital lobe: It has abitlity to recognize the objects. It is responsible for the vision system.

For the experiment, we need EEG singles from different lobes.

\section{EEG Systems}

The first International EEG congress was recognized, in 1947, that standard eletrodes placement method is needed for the EEG system (Oostenvelda et al., 2001). As a result 10-20 electrode system was introducted by H.H. Jasper in 1958. The 10-20 system, 21 electrodes are located, utilizes the scalp measurement according to the external landmarks relative to the cranial position on the head and their placement at distance of $10 \%$ and $20 \%$ of measured coronal, sagittal and circumference arcs between the nasion and inion. Electrodes are identified relative to their location on the scalp, for example, F, T, C, P and $\mathrm{O}$ stand for frontal, temporal, central, parietal, and occipital lobes. Odd numbers refer to the left side electrodes and even numbers refer to the right side electrodes. The ground electrode is placed at the neutral site on the head (midline forehead). The reference electrodes (A1 and A2) for the left and right respectively, are placed in active zone (ear lobes). The 10-10 system or International 10-20 system is an internationally recognized method to describe and apply the location of scalp electrodes in the context of an EEG test or experiment. This method was developed to ensure standardized reproducibility so that a subject's studies could be compared over time and subjects could be compared to each other. This system is based on the relationship between the location of an electrode and the underlying area of cerebral cortex. The "10" and "20" refer to the fact that the actual distances between adjacent electrodes are either $10 \%$ or $20 \%$ of the total front-back or right-left distance of the skull. The 10-10 system, known as the $10 \%$ system, is also introduced and endorsed by the standard of the American Electroencephalographic Society and the International Federation of Societies for Electroencephalography and Clinical Neurophysiology (Oostenvelda et al., 2001) where eletrodes' placement at distance of $10 \%$ and $10 \%$ of measured coronal, sagittal and circumference arcs between the nasion and inion. Another extended version that 10-5 system is allowing more than 300 electrode positions (Jurcak et al., 2007). Intracranial source of EEG signals is to facilite for the high density EEG applications.

\section{EEG Waves}

Typically the amplitude of the clinical EEG signal is around 10 to $100 \mu \mathrm{v}$ and the frequency range is 1 to $100 \mathrm{~Hz}$. EEG signal can be classified into five rhythms according to their frequency bands as explained below:

Delta waves ( $\boldsymbol{\delta})$ : The frequency range is less than $4 \mathrm{~Hz}$ with amplitude ranges from 20-200 $\mu \mathrm{v}$. It occurs during deep sleep, in infancy and in serious organic brain diseases (He, 2013). It can be recorded posteriorly in children and frontally in adults.

Theta waves $(\boldsymbol{\theta})$ : The frequency range for theta wave is 4 to $7 \mathrm{~Hz}$. It is prominently during sleep, emotional stress, and arousal in children and adults. It is recorded from the temporal and parietal region of the scalp with an amplitude range of 5-10 $\mu \mathrm{v}(\mathrm{He}, 2013)$.

Alpha waves $(\boldsymbol{\alpha})$ : It is rhythmic wave in healthy adults during wakefulness, relax and eye closed, occurs at frequency range of (8 to 13 ) $\mathrm{Hz}$ and normal voltage range is approximately $20-200 \mu \mathrm{v}$, and disappeared in pathological case like coma or sleep.

Beta waves ( $\beta$ ): The frequency range of Beta waves is $13-30 \mathrm{~Hz}$, while their amplitudes are lower, in the range of 5-10 $\mu \mathrm{v}$. It appears in case of extra excitation of the central nervous system, 
increase in attention and vigilance, it replaces the alpha rhythm during cognitive impairment. It can be recorded from the parietal and frontal region of the scalp.

Gamma waves $(\gamma)$ : Frequency ranges from 30 to $100 \mathrm{~Hz}$. It is recorded from the somatosensory cortex in the case of cross model sensory processing, during short term memory to recognize objects, sounds, tactile sensation and in the pathological case due to cognitive decline particularly when it related to $\theta$ band.

The main objective to seizure prediction/detection is able to timely identify the arrival of epileptic seizure in a fully automated way. The system architecture adopted such prediction/detection purposes are schematized in Fig. 4 and Fig. 5. Note that the detection technique follows the stages that are preprocessing, feature extraction, feature selection, and classification where as prediction has more two stages that are regularization and decision function. The concise description of the process diagram of detection and prediction are as follows:

\section{Pre-processing}

A pre-processing step is exploited in order to eliminate the influence of disturbance (i.e. artifact) of EEG signals. The artifact can be divided into two parts; one is physiological artifact that raised from the body and another is non-physiological artifact that comes from environment and instruments. There is several type of physiological artifact such as muscle artifact, pulse artifact and eye blinking artifact. The non-physiological artifact is power line artifact and sweat artifact (i.e. water, minerals, and lactate so on).

The details physiological artifacts are described below:

Muscle activity: It is characterized by surges in high frequency activity generated by neck and facial muscles in case of seizure, muscle contraction or movement due to increase muscle tone (Núñez, 2010).

Eye blinking artifact: It is generated by vertical movement of the eyes, its frequency concentrate in theta band and it produced around the eye electrodes FP1, FP2, F3 and F4 with high amplitude that corrupted EEG data on these electrodes. An eye blink is from 200 to $400 \mathrm{~ms}$ and it has an electrical magnitude more than 10 times that of cortical signals (Núñez, 2010).

Pulse: The pulse artifact is intorduced from a heartbeat of a patient (Oh, 2014). It can be detected in the occipital channels due to expansion and contraction of the blood vessels which is caused voltage change in to the recording.

The details non-physiological artifacts are describe below:

Power line artifact: The source of noise is acquired from the A/C power supplies $50 \mathrm{~Hz}(60 \mathrm{~Hz})$. It contributes all the channels due to its high amplitude value and noise is much higher than interested signals (Núñez, 2010).

Sweat artifact: This artifact occurs due to the sweat contents such as water, minerals, lactate and urea that cause the change in the electrode impedance and produces unstable baseline (Núñez, 2010).

Notch filter can be used to remove line noise interference. Wavelet transform is an efficient denoising technique that is introduced the non-linear and non-stationary EEG signals (Krishnaveni et al., 2006). Blind signal separation techniques based on principle component analysis (PCA) and independent component analysis (ICA) approach can separate unknown mixture of the signals (Nait-Ali, 2009). EMD has powerful nature to analysis non-stationary and non-liner EEG signals that achieve an effective approach to remove power line interference noise and other high frequency noise (Molla et al., 2010).

Another popular artifact removing technique, adaptive noise canceller (ANC) used to remove noise and artifact from the EEG signal (Olguin et al., 2005). An ANC system where $d(n)$ is the primary measurement containing signal $s(n)$ plus noise $v_{0}(n)$. The reference input $x(n)$ contains noise $v_{l}(n)$, which is correlated with $v_{0}(n)$ but not with $s(n)$. Hence, will the error signal $e(n)$ approximate the desired signal $s(n),(e(n)=s(n))$. Adaptive filter is used to adapt and remove ocular artifacts. 
Other statistical approaches that used to separate noise in EEG signal are the canonical correlation analysis (CCA), and matching pursuit (MP) method.

CCA is a statistical method used to use the remove muscle artifact from the EEG signals (Wim De et al., 2006). It is applied to seperate the muscle sources from brain activity sources due to relative muscle activity source is comparable with brain activity.

\section{Feature Extraction}

For the sake of classification, relevant features needed to be extracted from EEG signals. It is important to use real time classification techniques for prediction and detection purposes for actual applications. Moreover, computational burden should be reduced and false positive rate should be lowered for sensitivity while preserving high prediction ability. EMD, SVD, and DWT may be good techniques for the feature extraction of EEG signals as EEG signals has non-linear nature. The fundamental part of the Hilbert-Huang transformation (HHT) (Huang et al., 2006) is EMD method in which any linear/non-linear dataset is represented as a finite and small number of components called IMFs. This decomposition technique depends on local characteristics of dataset instead of pre-defined basis functions. Therefore, it is highly efficient and adaptive. In general, SVD is a powerful tool in linear algebra and has been extensively applied to signal processing, statistical analysis and mathematical modelling. Nonlinear SVD is an extension of SVD for both the qualitative detection and quantitative determination of nonlinearity in a time series. SVD can be considered as a generalization of the spectral decomposition of square matrices, to analyze rectangular matrices. SVD decomposes a rectangular matrix into three simple matrices: two orthogonal matrices and one diagonal matrix (non-zero singular value). The non-zero-singular values of SVD are the square roots of the non-zero eigenvalues. Non-zero-singular values can be used for distinguish features for EEG classification as they have distributed frequencies in higher order to lower order. In DWT, it is a wavelet transform for which the wavelets are discretely sampled. A key advantage is that it captures both frequency and location information (location in time). DWT works based on multi-level where each signal is decomposed into several scales and each scale provides a particular coarseness of signal. Each phase of decomposition of a signal is composed of two down samplers by 2 and two digital filters. In each phase, high pass filter which serves as the discrete mother wavelet, and low pass filter which acts as the mirror version of the corresponding. The downsampled outputs of the first low-pass and high-pass filters supply the approximation and detail information respectively. The first approximation is further decomposed and the procedure is continued. H. Adeli et al.,2013 mentioned that the Daubechies order 4-wavelet (db4) is the most suitable for EEG signal analysis. A EEG signal can be decomposed into different frequency bands such as gamma, beta, alpha, theta, and delta through DWT. Among the frequency bands, the gamma band is related to seizure as it carries more information than the other bands see in Fig. 6.

\section{Feature selection}

Feature selection is a technique that removes the irrelevant feature from the feature set and selects the most relevant one. PCA and ICA can be used for dimensionality reduction of the features (Naeem et al., 2009). PCA is based on an eigenvalue decompostion of the covarience matrix. It is a statistical procudure that usally uses an orthogonal transformation tehcnique to convert a set of observations of correlated variables into values of linearly uncorrelated variables that is called principle components. The priciple components is less or equal of original values. ICA is a computational method to reduce the additive subcomponents of the signals and retrive the original EEG signals. 

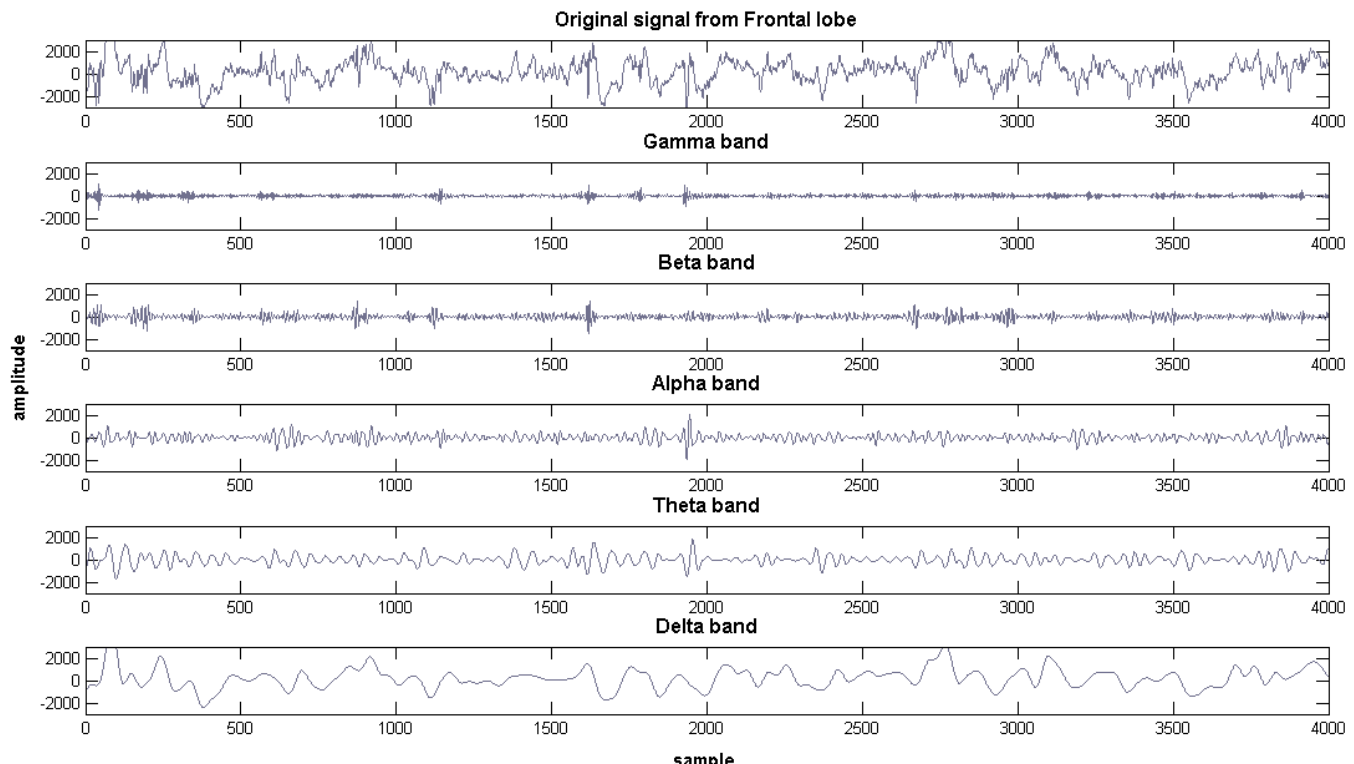

Figure 6. Different frequency band after applying DWT decomposed technique in preictal signal of Frontal lobe signal (patient one, 7th data block, and channel one).

\section{Classification}

The goal of a classifier is to find patients states such as preictal/ictal (Class 1) and interictal (Class 2) using machine learning approaches with cross-validation. The challenge is to find the mapping that generalized from training sets and unseen test sets. For the cross-validation, data are partitioned into training set and test set. SVM (Vapnik, 1995) is a good classifier for EEG signals classification. It can minimize the operational error and maximize the margin hyperplane, as a result it will maximize the classification performance. Moreove, there are several classifiers exist to classify feature vectors of EEG signals. Different classifiers are explained below:

LS-SVM: Let us consider binary classification problem with the training set ${ }^{\left\{x_{i}\right\}_{i=1}^{N}}$, along with corresponding targets $\left\{y_{i}\right\}_{i=1}^{N}$ where $x_{i} \in R^{n}$ and ${ }^{y_{i}=\{1,-1\}, i=1, \ldots N}$. Assume there exists a linear separable hyperplane $f(w, b)=\|w\|^{2} / 2$ such that

$w^{T} x_{i}+b \geq 1 \quad \forall_{i}: y_{i}=1$

$w^{T} x_{i}+b \leq-1 \quad \forall_{i}: y_{i}=-1$

where the decision function is:

$f(x)=\operatorname{sign}\left[w^{T} g(x)+b\right]$

where $w$ is the weight vector and $g(x)$ is the mapping function of $x$ and $b$ is a bias.

The optimization problem of LS-SVM can be solved in the following way:

$\min _{w, b, \xi} \frac{1}{2}\|w\|^{2}+\frac{C}{2} \sum_{i=1}^{N} \xi_{i}^{2}$

subject to $y_{i}\left(\left(w^{T} x_{i}\right)+b\right)=1-\xi_{i}, i=1 \ldots N$.

where ${ }^{\xi_{i}}$ are the slack variables, it represents the upper bound training error and $\mathrm{C}>0$ trades off margin size and training error.

Lagrange multipliers ${ }^{\alpha_{i}}$ can be introducing: 
$\min _{w, b, \xi, \alpha} \frac{1}{2}\|w\|^{2}+\frac{C}{2} \sum_{i=1}^{N} \xi_{i}^{2}-\sum_{i-1}^{N} \alpha_{i}\left\{1-y_{i}\left[w^{T} g\left(x_{i}\right)+b\right]-\xi_{i}\right\}$

subject to $\alpha_{i} \geq 0 ; i=1 \ldots N$.

For non-linear problem the kernel function is introduced in the above problem. Therefore, the decision function can be defined:

$f(x)=\operatorname{sign}\left[\sum_{i-1}^{N} \alpha_{i} y_{i} K\left(x, x_{i}\right)+b\right]$

where $K\left(x, x_{i}\right)$ is a kernel function.

Artificial Neural Network (ANN): It is non-linear classifier that has highly interconneting processing elements in the computing system (Subasi et al., 2005). It mimics the human brain activity in solving certain problems. The architecture of ANN is based on a set of nodes that are in input layer, output layer and hidden layer. The input layer that contained the input variables, and the output layer that achieves the solution to the problem in case of linear problems, an the hidden layer was employerd to handle the problem during the training process for nonlinear and complex behavior. The features of the are fed into ANN input for output observation. If in case the output is incorrect, then the training algorithm will modify the internal weight to minimize the difference between the desired and the actual output that is increase the accuracy. This training is continued until the network reaches a steady state.

Linear Discriminant Analysis (LDA): It is an optimal classifier that can be used to classify preictal/ictal and interictal EEG signals with low computational requirement. LDA evaluates the input by calculating the distance between a new sample and the mean of the training data sample in each class weighted by their covariance matrices. It tries to identify a hyperplane to separate features in the feature space in order to separate them into several classes (Lotte et al., 2007).

K- Nearest Neighbor (KNN): K- Nearest Neighbor classifier (KNN) is the simple and robust classifier with high performance results for non-linear data like EEG signals. The KNN is to determine the distace function to cluster the features into different group. This algorithm will identify the best features among the training set and weight it as a category candidate. The distance is calculated from the features vectors among the K-nearest neighbors by one of these approaches: Euclidean distance, Mahalanobis distance, Minkowiski distance, and Manhattan distance etc.

Parvez et al., 2013 has classified ictal and interictal EEG signals. The classification performance is evaluated using the receiver operating curve (ROC) shown in Fig. 7 and it visual classification result shown in Fig. 8.

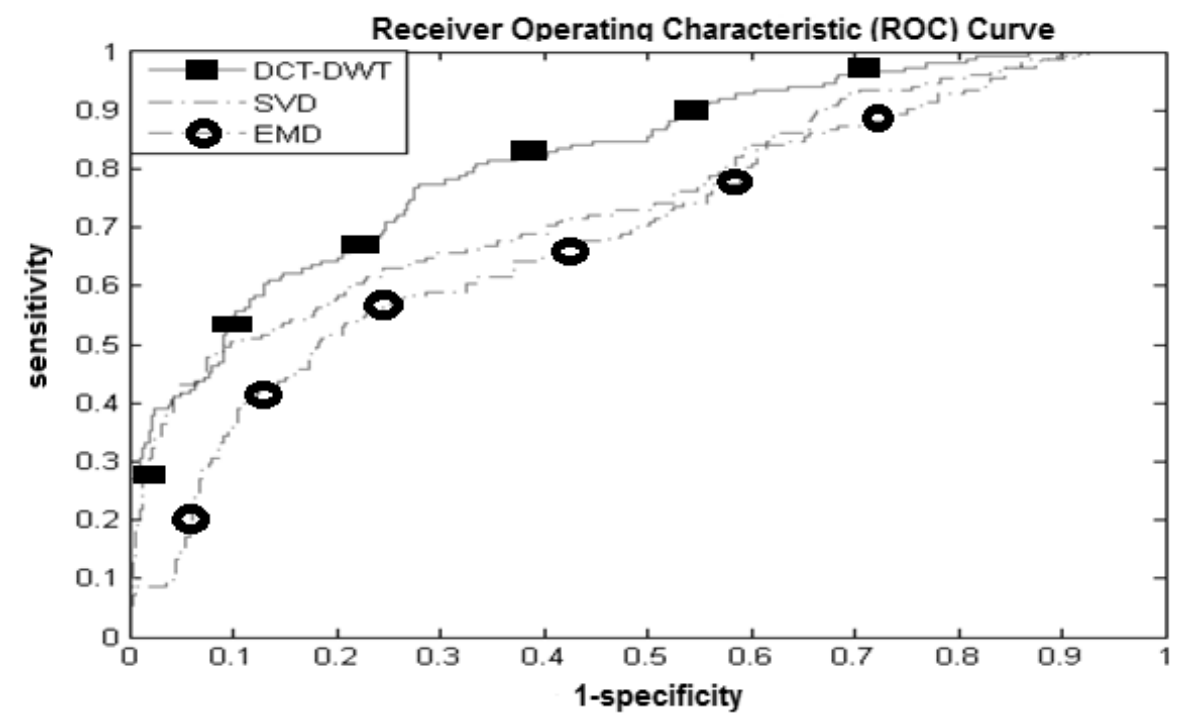

Figure 7. The receiver operating characteristics (ROC) curve of third subset of training and testing EEG signals using LS-SVM with RBF kernel from Frontal lobe (Parvez et al., 2013). 


\section{Regularization of Classification Outcome for Prediction}

Pre-processing, features extraction, features selection, and classification are the steps for detection. For prediction we need more steps such as regularization and decision function. Regularization is dealing with real classification data that is noisy. The noisy classification data is negatively affecting the seizure prediction techniques. Therefore, we need some regularization approach to overcome this problem. According to the non-linear nature of EEG signals, extended kalman filter (Lefebvre et al., 2001) or particle filter (Driessen et al., 2005) could be good practise to properly use of regularization approach as both filters are widely used for solving non-linear state estimation problems. In seizure prediction, we can apply kalman and particle filter for regularization of the over-fitting classification models for better classification results and eventually they reduce the false positive rate.

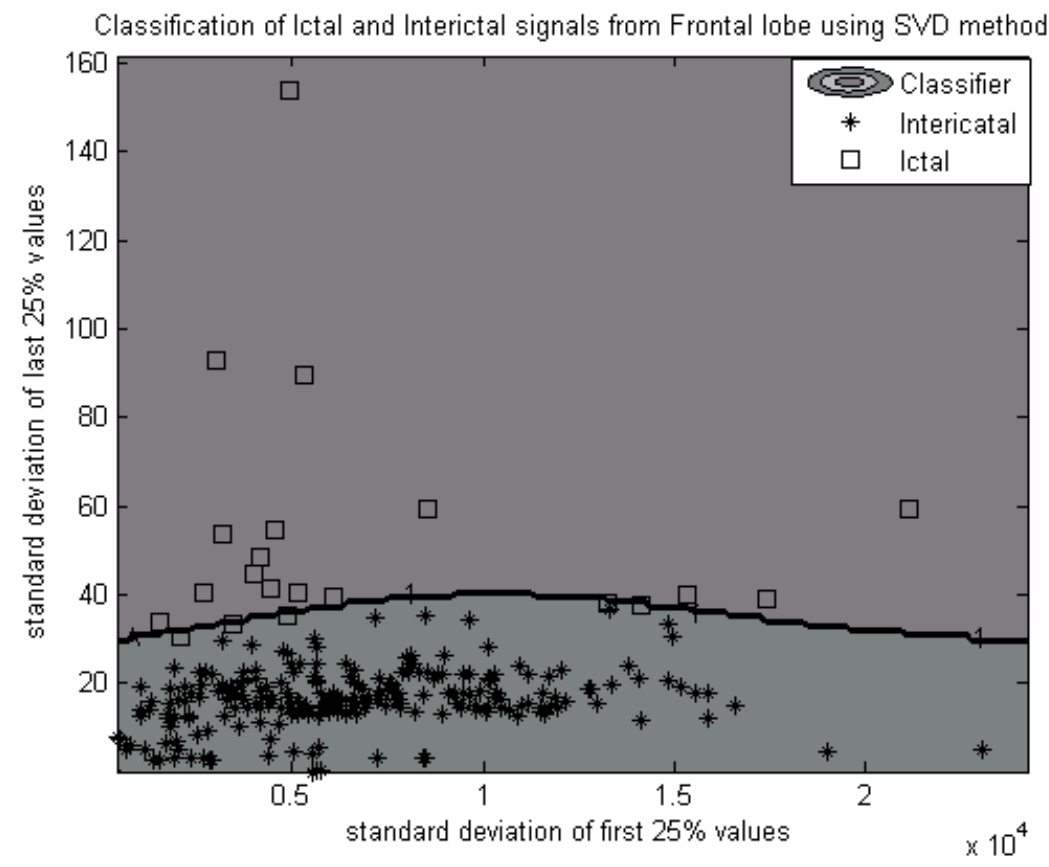

Figure 8 . Classification of Ictal and Interictal signals from Frontal lobe for the third subset of training and testing using the proposed method based on SVD (Parvez et al., 2013).

\section{Decision Function}

More accurate classification output can be obtained after regularization of classification results. However, a stand alone classification output of an EEG signal (or a part of an EEG signal) might not provide accurate predicted results; thus, sometimes a decision function can be formulated based on the combined classification output of different time-window of an EEG signal or a number of EEG signals in different channels. For the seizure prediction a number of criteria (such as accuracy, sensitivity, and specificity) are used to verify the classification outcome. Accuracy is determined as an overall performance measurement; however, sensitivity is determined from preictal signals and specificity from interictal signals. The accuracy, sensitivity and specificity are defined as follows where $T P$ is true positive, $T N$ is true negative, $F P$ is false positive, and $F N$ is false negative:

Accuracy $=\frac{T P+T N}{T P+T N+F P+F N} \times 100$ 
Sensitivity $=\frac{T P}{T P+F N} \times 100$

Specificity $=\frac{T N}{T N+F P} \times 100$

The perfomance of epileptic seizure prediction can be measured by the prediction accuracy (PA), false prediction rate (FPR), and mean prediction time (MPT). The three indicators are defined as:

$$
\begin{aligned}
& P A=(N s / N a) * 100 \\
& F P R=N f / N t \\
& M P T=T a / N s
\end{aligned}
$$

where $N s$ is the number of correctly predicted seizures, $N a$ is the total number of seizures, $N f$ is the number of inaccurately predicted seizures, $N t$ is the total time of EEG signals, $T a$ is the total amount of time that is predicted successfully.

In the window system decision function formulation, sensitivity and specificity are computed based on seizure prediction threshold with a seizure prediction window. Whenever, summation of all classification output in a given window is greater than seizure prediction threshold then the seizure prediction is triggered. If a seizure takes place during the specific time interval as indicated by the decision function then the seizure is correctly predicted. Moreover, kalman filter can be used to regularize the features to correctly seizure predition ( Chisci et al., 2010).

A detail performance analysis is given in the Table 2 by two state-of-the-art methods using a benchmark dataset where EEG signals are captured for 21 patients in 24 hours duration. The table shows that none of the algorithms provide $100 \%$ prediction accuracy for all patients with low false alarm rate. Thus, still there is a scope to perform research to improve the prediction accuracy as well as to reduce the false alarm rate.

\section{FUTURE RESEARCH DIRECTIONS}

We need an automated system to predict and detect EEG signals for different reasons:

- Detecting sophisticated changes in EEG signals correctly through manual process is a difficult task.

- Continuous monitoring of EEG signals for day to day is impossible by a person or a number of people.

- Having a qualified person who can diagnose the signals about a probable seizure is very costly.

Therefore, we have to analyse sophisticated changes of EEG signals for different dataset for more accuracy through automated or semi-automated system.

In the near future the following issues need to be addressed for correctly seizure detection and prediction:

- EEG capture machines may have more channels in future: we may need modern techniques which can exploit inter-channel correlation for better detection and prediction.

- Interferences of other signals on captured EEG signals: EEG signals may have interferences from other signals generated from portable electronics devices. For this, we may expect different line noise and artifacts. We may need different techniques to eliminate those unwanted noise through investigating the characteristics of those noises.

- Wireless signals and wired signals: EEG signals can be captured through wired and wireless devices. We may need to investigate whether the characteristics or noise are different in those signals. 
TABLE 2

PATIENTS DETAILS AND PREDICTED SEIZURE USING THE PHASE CORRELATION METHOD.

\begin{tabular}{|c|c|c|c|c|c|c|c|c|c|c|c|}
\hline \multirow{2}{*}{$\begin{array}{c}\text { Patien } \\
t\end{array}$} & \multirow{2}{*}{$\begin{array}{c}\mathrm{Se} \\
\mathbf{x}\end{array}$} & \multirow{2}{*}{ Age } & \multirow{2}{*}{$\begin{array}{c}\text { Seizure } \\
\text { Type }\end{array}$} & \multirow{2}{*}{$\mathrm{H} / \mathrm{NC}$} & \multirow{2}{*}{$\begin{array}{c}\text { Electrode } \\
\mathrm{s}\end{array}$} & \multirow{2}{*}{$\begin{array}{c}\text { Brain } \\
\text { Location } \\
\text { (Lobe) }\end{array}$} & \multirow{2}{*}{$\begin{array}{c}\text { Total } \\
\text { Seizure } \\
\text { s }\end{array}$} & \multicolumn{2}{|c|}{$\begin{array}{l}\text { Chisci, et } \\
\text { al., } 2010\end{array}$} & \multicolumn{2}{|c|}{$\begin{array}{l}\text { Park et al. } \\
2011\end{array}$} \\
\hline & & & & & & & & $\begin{array}{l}\text { PA } \\
(\%)\end{array}$ & FA & $\begin{array}{l}\text { PA } \\
(\%)\end{array}$ & FA \\
\hline 1 & $\mathrm{~F}$ & 15 & $\mathrm{SP}, \mathrm{CP}$ & $\mathrm{NC}$ & $\mathrm{g}, \mathrm{s}$ & Frontal & 4 & 100 & 0 & 100 & 1 \\
\hline 2 & $\mathrm{M}$ & 38 & $\begin{array}{l}\mathrm{SP}, \mathrm{CP}, \mathrm{GT} \\
\mathrm{C}\end{array}$ & $\mathrm{H}$ & $\mathrm{d}$ & Temporal & 3 & - & - & - & - \\
\hline 3 & $\mathrm{M}$ & 14 & SP,CP & $\mathrm{NC}$ & $\mathrm{g}, \mathrm{s}$ & Frontal & 5 & 100 & 3 & 100 & 1 \\
\hline 4 & $\mathrm{~F}$ & 26 & $\begin{array}{l}\text { SP,CP, } \\
\text { GTC }\end{array}$ & $\mathrm{H}$ & $\mathrm{d}, \mathrm{g}, \mathrm{s}$ & Temporal & 5 & - & - & 100 & 1 \\
\hline 5 & $\mathrm{~F}$ & 16 & $\begin{array}{l}\text { SP,CP, } \\
\text { GTC }\end{array}$ & $\mathrm{NC}$ & $\mathrm{g}, \mathrm{s}$ & Frontal & 5 & 100 & 23 & 100 & 21 \\
\hline 6 & $\mathrm{~F}$ & 31 & CP, GTC & $\mathrm{H}$ & $\mathrm{d}, \mathrm{g}, \mathrm{s}$ & $\begin{array}{l}\text { Temporal/Oc } \\
\text { cipital }\end{array}$ & 3 & - & - & 100 & 1 \\
\hline 7 & $\mathrm{~F}$ & 42 & $\begin{array}{l}\text { SP,CP, } \\
\text { GTC }\end{array}$ & $\mathrm{H}$ & $\mathrm{d}$ & Temporal & 3 & - & - & 100 & 1 \\
\hline 8 & $\mathrm{~F}$ & 32 & $\mathrm{SP}, \mathrm{CP}$ & $\mathrm{NC}$ & $\mathrm{g}, \mathrm{s}$ & Frontal & 2 & - & - & - & - \\
\hline 9 & $\mathrm{M}$ & 44 & $\mathrm{CP}, \mathrm{GTC}$ & $\mathrm{NC}$ & $\mathrm{g}, \mathrm{s}$ & $\begin{array}{l}\text { Temporal } \\
\text { /Occipital }\end{array}$ & 5 & 100 & 3 & 100 & 4 \\
\hline 10 & $\mathrm{M}$ & 47 & $\begin{array}{l}\text { SP,CP, } \\
\text { GTC }\end{array}$ & $\mathrm{H}$ & $\mathrm{d}$ & Temporal & 5 & - & - & 100 & 3 \\
\hline 11 & $\mathrm{~F}$ & 10 & $\begin{array}{l}\text { SP,CP, } \\
\text { GTC }\end{array}$ & $\mathrm{NC}$ & $\mathrm{g}, \mathrm{s}$ & Parietal & 4 & 100 & 9 & 75 & 2 \\
\hline 12 & $\mathrm{~F}$ & 42 & $\begin{array}{l}\text { SP,CP, } \\
\text { GTC }\end{array}$ & $\mathrm{H}$ & $\mathrm{d}, \mathrm{g}, \mathrm{s}$ & Temporal & 4 & - & - & 100 & 1 \\
\hline 13 & $\mathrm{~F}$ & 22 & $\begin{array}{l}\text { SP,CP, } \\
\text { GTC }\end{array}$ & $\mathrm{H}$ & $\mathrm{d}, \mathrm{s}$ & $\begin{array}{l}\text { Temporal/Oc } \\
\text { cipital }\end{array}$ & 2 & - & - & - & - \\
\hline 14 & $\mathrm{~F}$ & 41 & $\mathrm{CP}, \mathrm{GTC}$ & $\mathrm{H}, \mathrm{NC}$ & $\mathrm{d}, \mathrm{s}$ & $\begin{array}{l}\text { Frontal/Tem } \\
\text { poral }\end{array}$ & 4 & - & - & 75 & 12 \\
\hline 15 & $\mathrm{M}$ & 31 & $\begin{array}{l}\text { SP,CP, } \\
\text { GTC }\end{array}$ & $\begin{array}{l}\mathrm{H}, \\
\mathrm{NC}\end{array}$ & $\mathrm{d}, \mathrm{s}$ & Temporal & 4 & - & - & 100 & 4 \\
\hline 16 & $\mathrm{~F}$ & 50 & $\begin{array}{l}\text { SP,CP, } \\
\text { GTC }\end{array}$ & $\mathrm{H}$ & $\mathrm{d}, \mathrm{s}$ & Temporal & 5 & - & - & 90 & 11 \\
\hline 17 & $\mathrm{M}$ & 28 & $\begin{array}{l}\text { SP,CP, } \\
\text { GTC }\end{array}$ & $\mathrm{NC}$ & $\mathrm{s}$ & Temporal & 5 & 100 & 10 & 100 & 1 \\
\hline 18 & $\mathrm{~F}$ & 25 & SP,CP & $\mathrm{NC}$ & $\mathrm{s}$ & Frontal & 5 & 100 & 17 & 100 & 1 \\
\hline 19 & $\mathrm{~F}$ & 28 & $\begin{array}{l}\text { SP,CP, } \\
\text { GTC }\end{array}$ & $\mathrm{NC}$ & $\mathrm{s}$ & Frontal & 4 & 100 & 25 & 75 & 24 \\
\hline 20 & $\mathrm{M}$ & 33 & $\begin{array}{l}\text { SP,CP, } \\
\text { GTC }\end{array}$ & $\mathrm{NC}$ & $\mathrm{d}, \mathrm{g}, \mathrm{s}$ & $\begin{array}{l}\text { Temporal/Pa } \\
\text { rietal }\end{array}$ & 5 & 100 & 0 & 80 & 16 \\
\hline 21 & $\mathrm{M}$ & 13 & $\mathrm{SP}, \mathrm{CP}$ & $\mathrm{NC}$ & $\mathrm{g}, \mathrm{s}$ & Temporal & 5 & - & - & 100 & 4 \\
\hline
\end{tabular}

$\mathrm{SP}=$ simple partial, $\mathrm{CP}=$ complex partial, $\mathrm{GTC}=$ generalized tonic-conic, $\mathrm{H}=$ hippocampal origin, $\mathrm{NC}=$ neocortical origin, $\mathrm{d}=\mathrm{depth}$ electrode, $\mathrm{g}=$ grid electrode, $\mathrm{s}=$ strip electrode, $\mathrm{FA}=$ False alarm, - indicated that experiment is not available for this patient

\section{CONCLUSION}

A reliable seizure prediction and detection system could lead to better treatment and safety for epileptic patients. Therefore, it is very important to detect seizure onset not only for treatment but also for preventing the seizure. Prediction and detection of seizures, by analysing preictal/ictal, and interictal EEG signals with good sensitivity and specificity, could put away a patient from the next seizure. Automatic prediction is necessary due to three reasons; correctly detection, continuous monitoring and reduce operational cost. In this chapter we have explained the current state of the arts on the different steps of the seizure detection and predictions with their limitations and advantages. We have also indicated futures 
challenges of the prediction and detection. The accuracy of the prediction and detection in real time EEG signals are below the expected level, thus, more research should be conducted for better accuracy.

\section{REFERENCES}

Adeli, H., Zhou, Z., \& Dadmehr, N. (2003). Analysis of EEG records in an epileptic patient using wavelet transform. Journal of Neuroscience Methods, 123, 69-87.

Amine Nait-Ali. (2009). Advanced Biosignal Processing.

Bajaj, V., \& Pachori, R. B. (2012). Classification of Seizure and Non-seizure EEG Signals using Empirical Mode Decomposition. IEEE Transaction on Information Technology in Biomedicine, 16(6), 1135-1142.

Chisci, L., Mavino, A., Perferi, G., Sciandrone, M., Anile, C., Colicchio, G., \& Fuggetta, F. (2010). Real-Time Epileptic Seizure Prediction Using AR Models and Support Vector Machines. IEEE Transactions on Biomedical Engineering, 57(5).

Craig, F., \& Robynne, B. (2013). How Your Brain Works, Retrieved October 05, 2013 from http://science.howstuffworks.com/life/inside-the-mind/human-brain/brain1.htm.

Dastidar, S. G., Adeli, H., \& Dadmehr, N. (2007). Mixed-band wavelet chaos-neural network methodology for epilepsy and epileptic seizure detection. IEEE Transaction on. Biomedical Engineering., 54 ( 9), 1545-1551.

Di, W., Zhihua, C.,. Ruifang, F., Guangyu, L., \& Tian, L. (2010). Study on human brain after consuming alcohol based on EEG signal. 3rd Int. Conference on Computer Science and Information Technology (ICCSIT), 1 5, 406 - 409.

Dorr, V.L., Caparos, M., Wendling, F., Vignal, J. P., and Wolf, D. (2007). Extraction of reproducible seizure patterns based on EEG scalp correlations. Biomedical Signal Processing and Control, 2(3), 154-162.

Driessen, H., \& Boers, Y. (2005). Efficient particle filter for jump Markov nonlinear systems. IEE Proceedings Journals \& Magazines Radar, Sonar and Navigation, 152 (5), 323 - 326.

Duman, F., Ozdemir, N., \& Yildirim, E. (2012). Patient Specific Seizure Prediction Algorithm Using HilbertHuang Transform," IEEE-EMBS International Conference on Biomedical and Health Informatics, Hong Kong and Shenzhen, China.

Bonn University Epilepsy dataset, Retrieved April 28, 2012 from http://epileptologiebonn.de/cms/front content.php?idcat=193\&lang=3\&changelang=3.

Epilepsy Australia: Epilepsy Information. (2014.) http://www.epilepsyaustralia.net/Epilepsy Information/Epilepsy explained/Epilepsy explained.aspx

Epilepsy Center of the University Hospital of Freiburg Epilepsy dataset, Retrieved July 20,2013 from http://epilepsy.uni-freiburg.de/freiburg-seizure-prediction-project/eeg-database.

Esteller, R., Echauz, J. and Tcheng, T. (2004). Comparison of line length feature before and after brain electrical stimulation in epileptic patients. IEEE Annual International Conference in Engineering in Medicine and Biology Society, 2, 4710-4713.

Fisher, R. S., Van Emde Boas, W., Blume, W., Elger, C., Genton, P., Lee, P., \& Jr. Engel, J. (2005). Epileptic seizures and epilepsy: definitions proposed by the International League Against Epilepsy and the International Bureau for Epilepsy (IBE). Epilepsia, 46(4), 470-472.

Guttinger, M., Padrun, V., Pralong, W. F., and Boison, D. (2005). Seizure suppression and lack of adenosine A1 receptor desensitization after focal long-term delivery of adenosine by encapsulated myoblasts. Experimental Neurology, 193(1), 53-64.

He, B. (2013). Neural Engineering, Second Edition. Springer, 62-63.

Huang, H., \& Pan, J. (2006). Speech pitch determination based on Hilbert-Huang transform. Signal Processing, 86(4), 792-803.

Jurcak, V., Tsuzuki, D., \& Dan, I. (2007). 10/20, 10/10, and 10/5 systems revisited: Their validity as relative head-surface-based positioning systems. NeuroImage, 34(4), 1600-1611. 
Krishnaveni, V., Jayaraman, S., Anitha, L., \& Ramadoss, K. (2006) Removal of ocular artifacts from EEG using adaptive thresholding of 770 wavelet coefficients. Journal of Neural Engineering, 3(4), 338-346.

Lefebvre, T., Bruyninckx, H., \& Schutter, J. D. (2001). Kalman Filters for nonlinear systems: a comparison of performance. IEEE Transactions on Automatic Control.

Li, S., Zhou, W., Yuan,Q., \& Liu, Y., (2013). Seizure Prediction Using Spike Rate of Intracranial EEG. IEEE Transactions on Neural Systems and Rehabilitation Engineering, 21(6), 880-886.

Liang, S. F., Wang, H. C., \& Chang, W. L. (2010). Combination of EEG complexity and spectral analysis for epilepsy diagnosis and seizure detection. EURASIP Journal on Advances in Signal Processing.

Lotte, F., et al. (2007). A review of classification algorithms for EEG-based brain-computer interfaces. Journal of Neural Engineering, 4(2), 1-24.

Mirowski, P. W., LeCun, Y., Madhavan, D., \& Kuzniecky, R. (2008).Comparing SVM and convolutional networks for epileptic seizure prediction from intracranial EEG. IEEE Workshop Processing in Machine Learn. Signal Process (MLSP), Cancun, Mexico, Oct. 16-19, pp. 244-249.

Molla, K. I., Tanaka, T., Rutkowski, T. M., \& Cichocki, A. (2010). Separation of EOG artifacts from EEG signals using bivariate EMD. IEEE International Conference on Acoustics Speech and Signal Processing (ICASSP), $562-565$.

Naeem, M., Brunner, C., \& Pfurtscheller, G. (2009). Dimensionality Reduction and Channel Selection of Motor Imagery Electroencephalographic Data. Computational Intelligence and Neuroscience.

Netoff, T., Park, Y., \& Parhi, K. (2009). Seizure predition using cost-sensitive support vector machine. International conference of the IEEE EMBS, 3322-3325.

Neurology Applied. (2013). How Science is Bringing Music Instruction Back to Expressive Development Retrieved October 10, 2013 from http://www.thomasjwestmusic.com/neurologyapplied.htm.

Núñez, I.M.B. (2010). EEG Artifact Detection, 12-13.

Ocak, H. (2008). Optimal classification of epileptic seizures in EEG using wavelet analysis and genetic algorithm. Signal Processing, 88 (7), 1858-1867.

Oh, S.S., et al. (2014). A pulse artifact removal method considering artifact variations in the simultaneous recording of EEG and fMRI. Neuroscience Research, 81-82, 42-50.

Olguin, D., Bouchereau, F., \& Martınez, S. (2005). Adaptive Notch Filter for EEG Signals Based on the LMS Algorithm with Variable Step-Size Parameter. International Conference on Information Sciences and Systems.

Oostenvelda, R., \& Praamstra, P. (2001). The five percent electrode system for high-resolution EEG and ERP measurements. Clinical Neurophysiology, 112, 713-719.

Pachori, R. B. (2008). Discrimination between ictal and seizure-free EEG signals using empirical mode decomposition. Research Letters in Signal Processing.

Panda, R., Khobragade, P.S., Jambhule, P.D., Jengthe, S. N., Pal, P. R., \& Gandhi, T. K. (2010). Classification of EEG signal using wavelet transform and support vector machine for epileptic seizure diction. International Conference on Systems in Medicine and Biology (ICSMB), 405-408.

Park, Y., Luo, L., Parhi, K. K., \& Netoff, T. (2011). Seizure prediction with spectral power of EEG using cost-sensitive support vector machines. Epilepsia, 52(10), 1761-1770.

Parvez, M. Z., \& Paul, M. (2012). Features Extraction and Classification for Ictal and Interictal EEG Signals using EMD and DCT. International Conference on Computer and Information Technology (ICCIT). 132-137.

Parvez, M.Z., \& Paul, M. (2013). Classification of Ictal and Interictal EEG signals. IASTED conference on Biomedical Engineering, 791-031.

Parvez, M.Z., \& Paul, M. (2015). Detection of Pre-stage of Epileptic Seizure by Exploiting Temporal Correlation of EMD Decomposed EEG Signals. Journal of Medical and Bioengineering, 4(2), 110116.

Parvez, M.Z., \& Paul, M. (2014). Epileptic Seizure Detection by Analyzing EEG Signals using Different Transformation Techniques. Neurocomputing, 145, 190-200. 
Polat, K., \& Gunes, S. (2007). Classification of epileptiform EEG using a hybrid system based on decision tree classifier and fast Fourier transform. Applied Mathematics and Computation. 187( 2), 1017-1026.

Rasekhi, J., Mollaei, M. R. K., Bandarabadi, M., Teixeira, C. A., \& Dourado, A. (2013). Preprocessing effects of 22 linear univariate features on the performance of seizure prediction methods. Journal of Neuroscience Methods, 217, 9-16.

Rosso, O. A., Blanco, S. and Rabinowicz, A. (2003). Wavelet analysis of generalized tonic-clonic epileptic seizures. Signal Processing, 83 (6), 1275-1289.

Sanei, S. and Chambers, J.A. (2007). EEG Signal Processing. NewYork: Wiley.

Santaniello, S., Burns, S. P., Golby, A. J., Singer, J. M., Anderson, W. S. and Sarma, S. V. (2011). Quickest detection of drug-resistant seizures: An optimal control approach. Epilepsy \& Behavior,. 22(2011), S49-S60.

Schelter, B. , Timmer, J., \& Schulze-Bonhage, A. (2008), Seizure Prediction in Epilepsy: From Basic Mechanisms to Clinical Applications. NewYork: Wiley-VCH.

Scholler, S., Bosse, S., Treder, M. S., Blankertz, B., Curio, G., Müller, K.-R., \& Wiegand, T. (2012). Toward a Direct Measure of Video Quality Perception Using EEG. IEEE Transaction on Image Processing.

Soleymani, M., Pun, T., \& Pantic, M. (2012). Multi-Modal Emotion Recognition in Response to Videos. IEEE Transactions on Affective Computing

Stufflebeam, R. (2008). Neurons, Synapses, Action Potentials, and Neurotransmission. Consortium on Cognitive Science Instruction (CCSI), Retrieved October 17, 2013, from http://www.mind.ilstu.edu/curriculum/neurons intro/neurons intro.php.

Subasi, A., \& Erçelebi, E. (2005). Classification of EEG signals using neural network and logistic regression. Computer Methods and Programs in Biomedicine, 78(2), 87-99.

Tang, Y. and Durand, D.M. (2012). A tunable support vector machine assembly classifier for epileptic seizure detection. Expert Systems with Applications, 39 (4), 3925-3938.

Vapnik, V. (1995).The nature of statistical learning theory. Springer-Verlag, New-York.

Williamsona, J. R., Blissa, D. W., Brownea, D. W., \& Narayananb, J. T. (2012). Seizure prediction using EEG spatiotemporal correlation structure. Journal of Epilepsy \& Behavior, 25(2), 230-238.

WHO (World Health Organization). (2013). Epilepsy, Retrive October 10, 2013 from http://www.who.int/mediacentre/factsheets/fs999/en/.

Wim De, C., et al. (2006). Canonical Correlation Analysis Applied to Remove Muscle Artifacts From the Electroencephalogram. Biomedical Engineering, IEEE Transactions on, 53(12), 2583-2587.

Zhou, W., Liu , Y., Yuan, Q., \& Li, X. (2013) .Epileptic Seizure Detection Using Lacunarity and Bayesian Linear Discriminant Analysis in Intracranial EEG. IEEE Transactions on Biomedical Engineering, 60, 3375-3381.

\section{KEY TERMS \& DEFINITIONS}

Seizure: It is simply the medical condition or neurological disorder in which too many neurons are excited in the same time.

Epilepsy: It is a medical condition having spontaneously recurrent seizures.

Electroencephalogram (EEG): It is a record of ongoing electrical signal to represent the human brain activity.

Support Vector Machine (SVM): It is a potential methodology for solving problem in linear and nonlinear classification, function estimation, and kernel based learning methods.

Empirical Mode Decomposition (EMD): It can decompose a signal into finite number of intrinsic mode functions (IMFs), which are ordered from higher frequency components to lower frequency components.

Ictal: Actual seizure period.

Interictal: Period between two adjacent seizures.

Preictal: Prior to the actual seizure. 